\title{
Control del tamaño de microfibras magnéticas poliméricas durante el proceso de síntesis.
}

Size control of polymeric magnetic microfibers during the synthesis process

\author{
Rosa Maricela Ormaza Hugo ${ }^{1}$, Julio Rolando Coello Cabezas ${ }^{2}$ \& Edwin Fernando \\ Basantes Basantes ${ }^{3}$
}

Recibido: 10-10-2018 / Revisado: 17-11-2018 /Aceptado: 04-12-2018/ Publicado: 05-01-2019

\begin{abstract}
.
DOI: $\underline{\text { https://doi.org/10.33262/cienciadigital.v3i1.273 }}$

This paper describes an experimental study that aims to obtain polymeric agarose microfibers with magnetic inclusions, developed for biotechnological applications. The study contemplates the control of the dimensions (length and diameter) of the microfibers, the analysis of the way in which the NPMs of maghemite are incorporated in the polymeric hydrogel and the type of confinement they present. In the formation of the microfibres, the concentration of the aqueous phase in the continuous medium, the concentration of surfactant and the application of roughness with ferromagnetic coatings in the slide plates were tested. The methodological basis is based on the work of Aldana et al. (2015). The results obtained after the characterization through the use of optical techniques, showed that, with the modification of the confinement system (O-ring of $2 \mathrm{~mm}$ ) during the synthesis and the application of roughness (50\% HQ - Zeolite 50\%), the microfibers had diameters between 35 and $55 \mathrm{~nm}$ and lengths that reached a ratio of up to $88.5 \%$ with the thickness of the O-ring.
\end{abstract}

Keywords: Nanoparticles, Polymer, Agarose, Maghemite, Microfibers.

1 Escuela Superior Politécnica de Chimborazo, Panamericana sur Km 1 1/2. Riobamba, Ecuador. rormaza@espoch.edu.ec

${ }^{2}$ Escuela Superior Politécnica de Chimborazo, Facultad de Recursos Naturales. Riobamba, Ecuador. julio.coello@espoch.edu.ec

3 Escuela Superior Politécnica de Chimborazo, Facultad de Recursos Naturales. Riobamba, Ecuador. edw_bas@yahoo.es 


\section{Resumen.}

Este trabajo describe un estudio experimental que tiene como objetivo la obtención de microfibras poliméricas de agarosa con inclusiones magnéticas, desarrolladas para aplicaciones biotecnológicas. El estudio contempla, el control de las dimensiones (longitud y diámetro) de las microfibras, el análisis de la forma en la que las NPMs de maghemita se incorporan en el hidrogel polimérico y el tipo de confinamiento que presentan. En la formación de las microfibras se ensayó con, la concentración de la fase acuosa en el medio continuo, la concentración de surfactante y la aplicación de rugosidad con recubrimientos ferromagnéticos en las placas portaobjetos. La base metodológica se sustenta en el trabajo de Aldana y col. (2015). Los resultados obtenidos luego de la caracterización mediante el uso de técnicas ópticas, evidenciaron que, con la modificación del sistema de confinamiento (junta tórica de $2 \mathrm{~mm}$ ) durante la síntesis y la aplicación de rugosidad (50\% HQ - Zeolita 50\%), las microfibras presentaron diámetros entre 35 y $55 \mathrm{~nm}$ y longitudes que alcanzaron una relación de hasta un 88,5 \% con el grosor de la junta tórica.

Palabras claves: nanopartículas, polímero, agarosa, maghemita, microfibras.

\section{Introducción.}

En los últimos años han proliferado las aplicaciones biotecnológicas en las que se emplean suspensiones coloidales en base a nanopartículas magnéticas (NPMs). Esto se debe fundamentalmente a la introducción de una nueva variable de control externo (su magnetismo) (Tartaj, Morales, González-Carreño, Veintemillas-Verdaguer, \& Serna, 2005). Por lo general, estas partículas son de óxido de hierro, cuasi-esféricas y de tamaño superparamagnético; constituyendo por tanto ferrofluidos (García-Cerda, RodríguezFernández, Betancourt-Galindo, Saldívar-Guerrero, \& Torres-Torres, 2003). En la mayoría de las aplicaciones se requiere que las NPMs tengan un recubrimiento superficial que las haga biocompatibles y no tóxicas (Lin, y otros, 2012). Por ello, están siendo incorporadas en el seno de redes poliméricas, generalmente de agarosa (Bhat, Tripathi, \& Kumar, 2010), (Zamora Mora, Soares, Echeverria, Hernández , \& Mijangos, 2015).

La agarosa es un polisacárido formado por unidades repetitivas de agarobiosas ((1-3)- $\beta$-Dgalactosa y (1-4)-ligado 3,6-anhydro- $\alpha$-1-galactosa). Es extraída de algas marinas y se la considera como un polisacárido neutro que forma geles termorreversibles al enfriar (Zamora Mora, Soares, Echeverria, Hernández , \& Mijangos, 2015). Este elemento presenta varias ventajas sobre otros polímeros naturales: es fácilmente maleable en diferentes formas y, puede ser modificada químicamente mediante el uso de proteínas, péptidos u otros agentes, lo cual puede alterar sus propiedades biocompatibles, y en ciertos casos sus propiedades mecánicas. Sin duda su característica más significativa es que, a diferencia de otros polímeros, la agarosa es no biodegradable y por lo tanto puede ser utilizada a largo plazo, en 
caso que esto sea necesario (Dias, Hussain, Marcos, \& Roque, 2011), (Lewitus, y otros, 2011) , (Estrada Guerrero, Lemus Torres, Mendoza Anaya, \& Rodriguez Lugo, 2010). Además, es soluble en agua a temperaturas superiores a $\operatorname{los} 65^{\circ} \mathrm{C}$, dependiendo del grado de sustituciones hidroxietílicas de sus cadenas laterales. Estas sustituciones se pueden modificar para que la temperatura de gelificación varíe entre los 17 y los $45^{\circ} \mathrm{C}$ (Ruiz Estrada, 2004).

Teniendo como antecedente principal el trabajo realizado por Aldana y col. (Aldana, Vereda, Hidalgo-Alvarez, \& de Vicente, 2016), sobre el desarrollo de un mecanismo sencillo para la síntesis de microfibras magnéticas flexibles y, con el desarrollo de este trabajo, se pretende profundizar en el control de parámetros específicos de las microfibras, así como en el conocimiento de su estructura, mediante el uso de nuevos materiales y la variación de ciertos parámetros fisicoquímicos. El objetivo principal de este trabajo fue controlar las dimensiones (longitud y diámetro) de las microfibras magnéticas poliméricas.

\section{Metodología:}

Las microfibras poliméricas magnéticas, se prepararon siguiendo la metodología propuesta por Aldana y col. (Aldana, Vereda, Hidalgo-Alvarez, \& de Vicente, 2016). Esta consiste, en el auto ensamblado de microfibras en presencia de un campo magnético de la fase magnética de una dispersión coloidal (fase acuosa de una emulsión agua/aceite), lo que lleva a la formación de agregados lineales que logran ser permanentes mediante la formación de una red polimérica.

Con el objetivo de mejorar las características de las fibras generadas por Aldana y col.(2016), se realizaron cambios en la técnica. El cambio principal, consistió en sustituir el elemento en el cual se coloca la emulsión al someterla al campo magnético. Es decir, en el proceso de formación de las microfibras se empleó una estructura formada por 2 placas portaobjetos separadas por una junta tórica, las cuales están sujetadas mediante ligas elásticas que ayudan a mantenerlas unidas. Una vez concluida la homogeneización de la muestra, se colocó la emulsión en el sistema de placas-junta tórica, el cual previamente fue posicionado en presencia del campo magnético. El proceso de formación de las microfibras requirió de 4 minutos aproximadamente. Luego de este tiempo, se retiró la muestra y se la sometió a un proceso de gelificación (Ver Figura 1.). A continuación, se realizó el lavado, almacenamiento y posterior observación.

Figura 1: Esquema de síntesis con estructura de placas portaobjetos-junta tórica. 1. Preparación de disoluciones, 2. Emulsión, 3. Sistema placas-junta tórica en el campo magnético, 4. Colocación de emulsión y formación de fibras, 5. Gelificación en presencia de campo magnético, 6. Lavado y visualización de microfibras. 


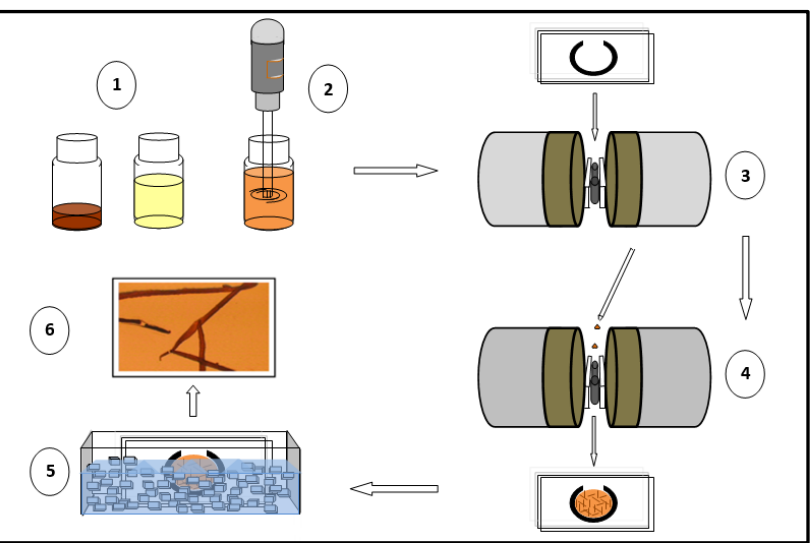

En la preparación de las disoluciones, previo el proceso de homogeneización, se consideró volúmenes de $250 \mu \mathrm{L}, 500 \mu \mathrm{L}$ y $1 \mathrm{~mL}$ de fase acuosa en medio continuo, siendo el de $500 \mu \mathrm{L}$, el volumen empleado en el proceso de síntesis por ser más efectivo. En el caso de la concentración de surfactante en el medio continuo, se realizaron pruebas tomando concentraciones de $0,10 \%, 0,12 \%$ y $0,15 \%$, llegando a determinarse que con el $0,15 \%$ se obtienen mejores resultados dentro del proceso, siendo ésta la concentración utilizada.

En el confinamiento de las microfibras, inicialmente se trabajó con juntas tóricas de diverso grosor: 1,8; 2,0; 2,5 y 3,0 mm. Luego y según la respuesta lograda durante los ensayos en el proceso de formación y crecimiento, es decir, por la relación conseguida de la longitud de las microfibras de acuerdo al grosor de la junta tórica, se empleó para el proceso de síntesis, únicamente la junta tórica de $2 \mathrm{~mm}$ de grosor.

Con el objetivo de conseguir microfibras con longitudes similares al grosor de la junta tórica utilizada (confinamiento), se introdujeron niveles de rugosidad en las placas portaobjetos. Para esto, se empleó un elemento ferromagnético, hierro carbonilo y un elemento no ferromagnético, zeolita. Con esto, también se buscó determinar si la presencia de rugosidad evita la agregación lateral de las NPMs y permite obtener microfibras más finas, con longitudes más largas y estructuralmente homogéneas.

Para la caracterización de las microfibras se utilizó microscopía óptica y microscopia electrónica de transmisión (TEM).

\section{Resultados y discusión:}

La síntesis de microfibras inicial fue realizada según Aldana y col. (2016), esto es, utilizando tubos de ensayo, con el fin de comparar los resultados luego de implementar el sistema de placas portaobjetos y junta tórica. Los resultados obtenidos se presentan en la Tabla 1.

Tabla 1: Dimensiones de microfibras sintetizadas en tubos y placas.

Resultados de longitud y diámetro obtenidos al utilizar tubos de ensayo y placas conjunta tórica de diferente grosor. 
ISSN: 2602-8085

Vol. 3, $\mathrm{N}^{\circ} 1$, p. 107-117, enero - marzo, 2019

\begin{tabular}{lcccccc}
\hline \multirow{2}{*}{ MUESTRA } & \multicolumn{3}{c}{ LONGITUD } & \multicolumn{3}{c}{ DIÁMETRO } \\
\cline { 2 - 7 } & Media $(\boldsymbol{\mu m})$ & $\begin{array}{c}\text { Desv. Est. } \\
(\boldsymbol{\mu m})\end{array}$ & $\boldsymbol{\%}$ Desviación & Media $(\boldsymbol{\mu m})$ & $\begin{array}{c}\text { Desv. Est. } \\
(\boldsymbol{\mu m})\end{array}$ & \% Desviación \\
\hline tubo muestra 1 & 769,9 & 553,9 & $72 \%$ & 30,8 & 7,6 & $25 \%$ \\
\hline tubo muestra 3 & 594,9 & 319,7 & $54 \%$ & 33, & 9,0 & $27 \%$ \\
\hline tubo muestra 2 & 483,0 & 231,1 & $48 \%$ & 34,5 & 11,6 & $34 \%$ \\
\hline junta tórica 1,8 $\mathbf{~ m m}$ & 490,4 & 166,4 & $34 \%$ & 26,5 & 6,6 & $25 \%$ \\
\hline junta tórica 2,5 $\mathbf{~ m m}$ & 1083,1 & 407,0 & $38 \%$ & 61,4 & 16,7 & $27 \%$ \\
\hline junta tórica 3 $\mathbf{~ m m}$ & 1414,3 & 503,9 & $36 \%$ & 47,9 & 11,1 & $23 \%$ \\
\hline
\end{tabular}

Con la comparación de la medida de longitud de las microfibras resultantes de la síntesis con el uso del sistema de tubos de ensayo y el de placas-junta tórica, es claro que las obtenidas con el segundo sistema, presentan un nivel más bajo de desviación en la longitud, con porcentajes de 34, 38 y 36\%. Mientras que, con el empleo de los tubos los valores tienen 72, 54 y $48 \%$ de desviación. En las medidas del diámetro no se evidencia grandes variaciones. Con estos resultados, se establece que el cambio de estructura realizado es adecuado.

Una vez determinada la eficiencia del sistema placas-junta tórica y realizados los análisis manejando volúmenes de 250 y $500 \mu \mathrm{L}$, de la fase acuosa en el medio continuo, se obtuvieron microfibras con diámetros de 44,90; 43,54 y 47,89 $\mu \mathrm{m}$ para el caso de los $250 \mu \mathrm{L}$; con grosores de junta tórica de 1,8; 2,5 y $3 \mathrm{~mm}$., respectivamente. Mientras que, con $500 \mu \mathrm{L}$ se obtuvieron diámetros de 33,39; 43,2 y 46,3 $\mu \mathrm{m}$. Al utilizar $500 \mu \mathrm{L}$, los resultados de la medida de longitud de las fibras presentan mayor similitud con el grosor de las juntas tóricas. Entonces el volumen más adecuado de fase acuosa en el medio continuo fue el de $500 \mu \mathrm{L}$. Esto se evidencia en la Figura 2.

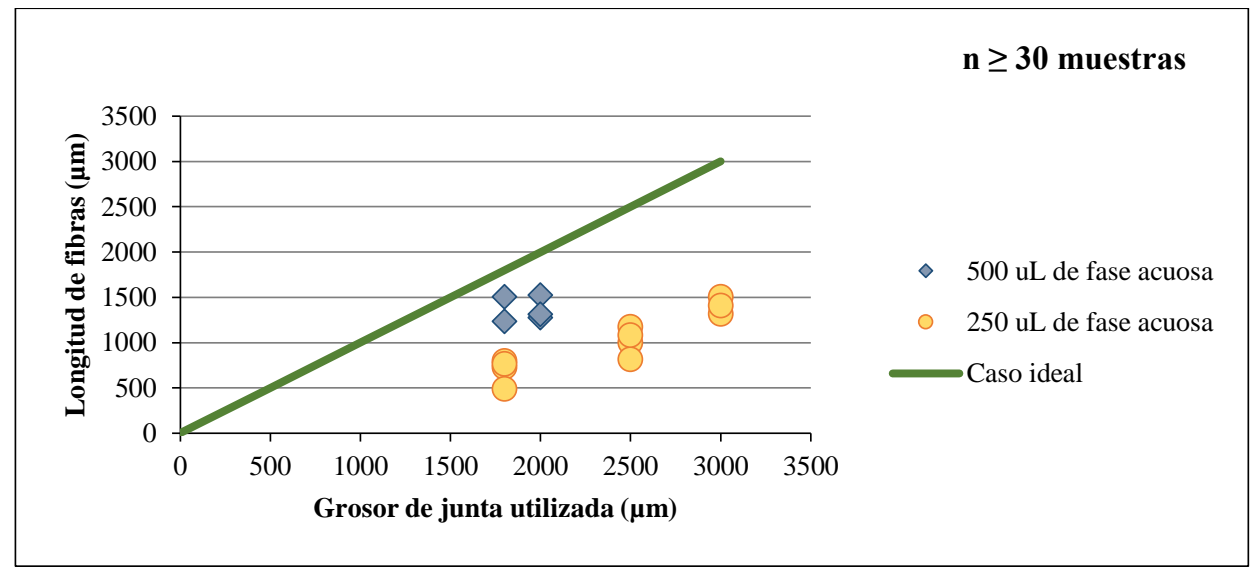

Figura 2. Longitud de fibras al utilizar 250 y $500 \mu \mathrm{L}$ de fase acuosa en medio continuo, comparadas con el caso ideal.

Los resultados para la concentración de $500 \mu \mathrm{L}$ determinan que la separación entre placas influye fuertemente en la longitud final de las fibras, en este caso la distribución estándar de 
las longitudes disminuye y la longitud promedio se acerca más a la separación entre placas, esto se debe a la mayor concentración de fase acuosa en la emulsión, que lleva a que las fibras formadas sean en general más largas y lleguen a contactar con las dos superficies de las placas porta objetos, logrando un confinamiento más eficiente de las fibras. Se puede decir que el diámetro de las fibras no depende de la separación entre placas, esto sugiere que el grosor de las fibras está determinado por el tamaño de gota de la emulsión.

Logrando una adecuada concentración de surfactante en el medio continuo se consiguió estabilizar de mejor manera la emulsión y se logró mejores propiedades en las microfibras resultantes. En ciertos casos, a bajas concentraciones se presentó la formación de agregados irregulares, y con concentraciones altas no se llegó a formar fibras. Se probó oncentraciones de $0,8 \%, 0,10 \%, 0,12 \%$ y $0,15 \%$ de poliglicerol polirricino oleato en aceite de girasol. Se pudo evidenciar que la concentración de $0,8 \%$, forma agregados muy grandes que no permiten el análisis. Con esto se determinó que la concentración de $0,15 \%$ presenta una mejor relación longitud-diámetro y un nivel más bajo de desviación, lo que es óptimo para el control de estos parámetros en las microfibras sintetizadas.

El desarrollo de este análisis, fue importante, puesto que el uso de surfactantes y su adecuada concentración en el medio continuo, permite obtener emulsiones estructuralmente más estables (Wulff-Pérez , Martín-Rodriguez, Gálvez-Ruiz, \& de Vicente, 2013 ), lo que se considera relevante al momento de formar microfibras a partir de emulsiones ferromagnéticas.

Sobre esto, Song y colaboradores (Song, King, Yoon, Cho, \& Jeong, Enhanced spinnability of narbon nanotube fibers by surfactant addition, 2014), mencionan que el empleo de surfactantes reduce la aglomeración de partículas de hierro durante una síntesis, y con ello se logra una mejor perfección cristalina y menor diámetro en las muestras obtenidas.

Los resultados de las fibras sintetizadas con la incorporación de rugosidad en las placas, se presenta en la Tabla 2. Al utilizar rugosidad de 50\% HQ-Zeoita se obtuvieron microfibras con un $88,5 \%$ de relación longitud-grosor de la junta, con desviación de 9,7\%, lo que nos permite determinar que la presencia de elementos con niveles magnéticos en las paredes de las placas, genera un efecto positivo al momento de la agregación y formación de las fibras. En general, se logra incrementar la agregación de las NPMs debido al aumento de polarización magnética que producen los elementos utilizados.

El diámetro presenta un valor medio de 41,29 $\mu \mathrm{m}$ con un 19,6\% de desviación, considerándose aceptable puesto que las dimensiones no difieren en gran medida con las obtenidas en las otras muestras.

Tabla 2. Dimensiones de microfibras preparadas en presencia de rugosidad. 
ISSN: 2602-8085

Vol. 3, N¹, p. 107-117, enero - marzo, 2019

\begin{tabular}{cccccccc}
\hline & \multicolumn{3}{c}{ Longitud } & & \multicolumn{2}{c}{ Diámetro } \\
\hline Muestra & $\begin{array}{c}\text { Media } \\
(\boldsymbol{\mu m})\end{array}$ & $\begin{array}{c}\text { Desv. } \\
\text { Est. }\end{array}$ & $\begin{array}{c}\text { \% de } \\
\text { variación }\end{array}$ & $\begin{array}{c}\text { Relación longitud- } \\
\text { grosor de junta tórica } \\
\mathbf{2} \mathbf{~ m m}\end{array}$ & $\begin{array}{c}\text { Media } \\
(\boldsymbol{\mu m})\end{array}$ & $\begin{array}{c}\text { Desv. } \\
\text { Est. }\end{array}$ & $\begin{array}{c}\text { \% de } \\
\text { variación }\end{array}$ \\
\hline $\mathbf{1 0 0 \%}$ HQ & 1439,9 & 208,2 & $14,5 \%$ & $72,0 \%$ & 44,7 & 8,2 & $18,3 \%$ \\
\hline $\mathbf{5 0 \%}$ HQ - Zeolita 50\% & 1770,0 & 171,5 & $9,7 \%$ & $88,5 \%$ & 41,3 & 8,1 & $19,6 \%$ \\
\hline $\mathbf{2 0 \%}$ HQ - Zeolita 80\% & 1618,4 & 197,1 & $12,2 \%$ & $80,9 \%$ & 45,9 & 9,0 & $19,7 \%$ \\
\hline Zeolita 100\% & 1597,8 & 152,7 & $9,6 \%$ & $79,9 \%$ & 30,2 & 5,4 & $17,8 \%$ \\
\hline
\end{tabular}

Las fibras no lograron obtener longitudes que se aproximen al 100\% del grosor de la junta tórica, lo cual se asocia a la variación de temperatura, lo que generó cambios en el volumen del gel de agarosa. Inicialmente las muestras se encuentran a $90{ }^{\circ} \mathrm{C}$, luego a temperatura ambiente mientras son sometidas al campo magnético y durante la gelificación llegan hasta los $0{ }^{\circ} \mathrm{C}$. Se examinó un gel de agarosa-agua y otro de agarosa-ferrofluido, obteniendo un cambio en su tamaño de $7,5 \%$ y $21.4 \%$ respectivamente. Con el análisis se puede asegurar que el gel se contrae al disminuir la temperatura del sistema, atribuyéndolo a la evaporación del agua superficial que se da a temperaturas entre 25 y $80^{\circ} \mathrm{C}$ (Cortés, Puig, Morales , \& Mendizábal, 2011).

Pudiendo decir que, debido a la naturaleza polimérica del hidrogel empleado (agarosa), las fibras se contraen cuando se baja la temperatura del sistema para forzar la gelificación del polímero, lo que representaría diferencias en torno al $15 \%$ en la longitud de las microfibras al momento de ser sintetizadas, este resultado se puede comparar al presentado por Zamora y col. (Zamora Mora, Soares, Echeverria, Hernández , \& Mijangos, 2015).

Las muestras fueron observadas con el uso de microscopía óptica, mientras que el estudio de la incorporación de las partículas de maghemita, se realizó mediante microscopía de transmisión (TEM). Con esta última, se analizó la distribución de las partículas de maghemita en el gel de agarosa. Las imágenes fueron tomadas con resolución de 100 y 200nm (Figura 3), donde podemos ver la distribución homogénea.

En base a estudio se determinó la distribución espacial y el tipo de confinamiento que presentan, las imágenes nos permiten evidenciar que la distribución de las partículas de maghemita en la red polimérica (agarosa) es homogénea, debido básicamente a la formación de enlaces entre las NPMs y los correspondientes grupos funcionales presentes en la superficie del gel (Ilg, 2013).

Figura 3. Distribución de maghemita en el gel de agarosa. a) 200nm, b) a 100nm. 
a)

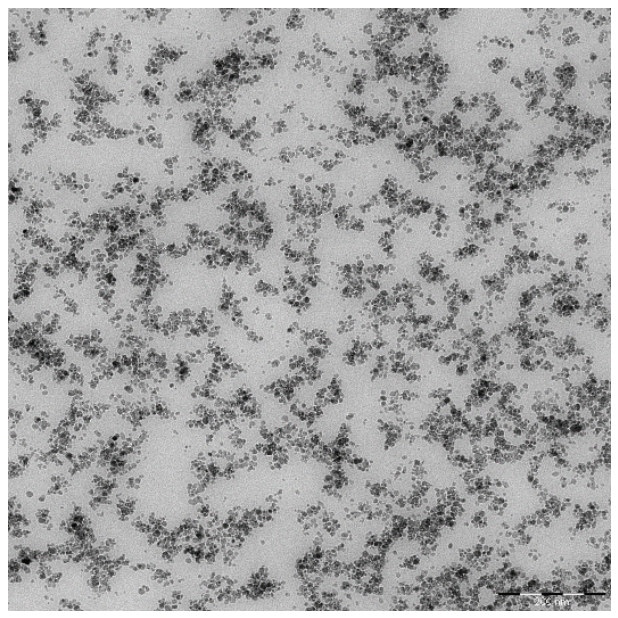

b)

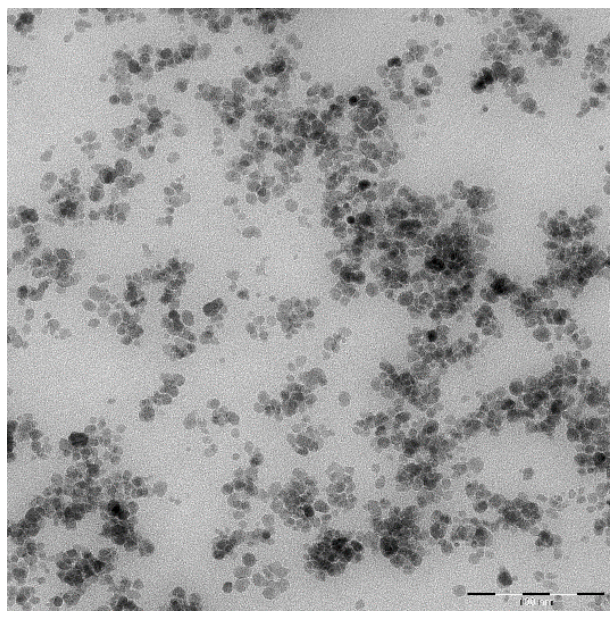

La microscopía óptica, permitió observas microfibras a 40X. Con esto se logra evidenciar la estructura de las mismas, las cuales presentan terminación aparentemente en punta según lo presentado en la figura 4.

Figura 4. Microfibras en microscopio óptico 40X.

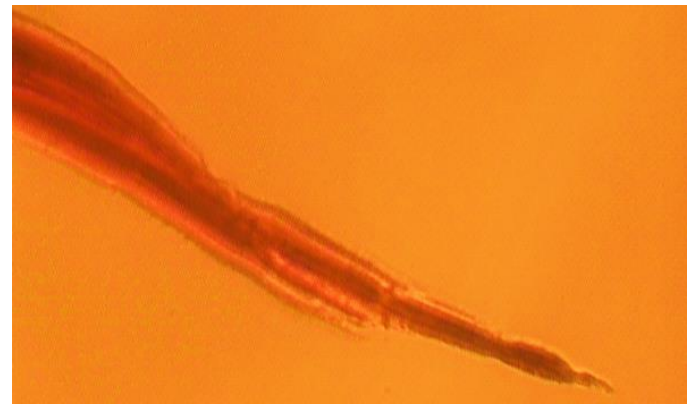

De manera general se puede decir que las microfibras obtenidas con dimensiones prácticamente controladas, presentan mejores propiedades en comparación con suspensiones magnéticas esféricas sintetizadas bajo las mismas condiciones. La forma alargada y sobre todo el hecho de mantener incrustadas NPMs (maghemita) en su superficie, puede aumentar las posibilidades de ser usadas en ámbitos biológicos (Bossis, Marins, Kuzhir, Volkova, \& Zubarev, 2015).

\section{Conclusiones}

- La elección de la estructura formada por placas portaobjetos y junta tórica para realizar el confinamiento del proceso de crecimiento de las microfibras, se considera acertada, pues hemos obtenido dimensiones más controladas. Por otro lado, es un sistema barato y sencillo, características importantes al momento de utilizarlo. 
- De manera general, y en base a los resultados obtenidos podemos decir que las microfibras presentan dimensiones prácticamente controladas, y sus propiedades han mejorado notablemente. La forma alargada y sobre todo el hecho de mantener incrustadas NPMs (maghemita) en su superficie, puede aumentar las posibilidades de ser usadas en diversos ámbitos (Lin, y otros, 2012; Bossis, Marins, Kuzhir, Volkova, \& Zubarev, 2015).

- Es importante realizar estudios futuros que permitan analizar mediante técnicas instrumentales más sofisticadas, los procesos de incorporación de las partículas de maghemita al hidrogel polimérico, su distribución espacial y el tipo de confinamiento que presentan.

\section{Agradecimientos}

- Es importante agradecer al Departamento de Física Aplicada de la Universidad de Granada, por permitir realizar el presente estudio.

\section{Referencias bibliográficas.}

Aldana, S., Vereda, F., Hidalgo-Alvarez, R., \& de Vicente, J. (2016). Facile synthesis of magnetic agarose microfibers by directed selfassembly. Polymer, 93, 61-64.

Bhat, S., Tripathi, A., \& Kumar, A. (2010). Supermacroprous chitosan-agarose-gelatin cryogels. in vitro characterization and in vivo assesment for cartilage tissue engineering. Journal of the Royal Society Interface, 1-15.

Bossis, G., Marins, J., Kuzhir, P., Volkova, O., \& Zubarev, A. (2015). Functionalized microfibers for field-responsive materials and biological applications. Journal of Intelligent Material Systems and Structures, 1-9.

Cortés, J., Puig, J., Morales , J., \& Mendizábal, E. (2011). Hidrogeles nanoestructurados termosensibles sintetizados mediante polimerización en microemulsión inversa. Revista Mexicana de Ingeniería Química., 10(3), 513-520.

Dias, A., Hussain, A., Marcos, A., \& Roque, A. (2011). A biotechnological perspective on the application of iron oxide magnetic colloids modified with polysaccharides. Biotechnology Advances 29, 29, 142-155.

Estrada Guerrero, R., Lemus Torres, D., Mendoza Anaya, D., \& Rodriguez Lugo, V. (2010). Hidrogeles poliméricos potencialmente aplicables en Agricultura. Revista Iberoamericana de Polímeros, 12(2), 76-87.

García-Cerda, L., Rodríguez-Fernández, O., Betancourt-Galindo, R., Saldívar-Guerrero, R., \& Torres-Torres, M. (2003). Síntesis y propiedades de ferrofluidos de magnetita. Superficies y Vacío., 16(1), 28-31. 
Ilg, P. (2013). Stimuli-responsive hydrogels cross-linked by magnetic nanoparticles. Soft Matter, 9, 3465-3468.

Lewitus, D., Branch, J., Smith, K., Callegari, G., Kohn, J., \& Neimark, A. (2011). Biohybrid carbon nanotube/agarose fibers for neural tissue engineering. Advanced Functional Materials, 21, 2624-2632.

Lin, Y.-S., Huang, K.-S., Yang, C.-H., Wang, C.-Y., Yang, Y.-S., Hsu, H.-C., . . Tsai, C.W. (2012). Microfluidic synthesis of microfibers for magnetic-responsive controlled drug release and cell culture. PLoS ONE, 7(3), 1-8.

Ruiz Estrada, G. (2004). Desarrollo de un Sistema de liberación de fármacos basado en nanopartículas magnéticas recubiertas con Polietilénglicol para el tratamiento de diferentes enfermedades. Madrid: Universidad Autónoma de Madrid. Departamento de Física Aplicada.

Song, J., King, S., Yoon, S., Cho, D., \& Jeong, Y. (2014). Enhanced spinnability of narbon nanotube fibers by surfactant addition. Fiberes and Polymers, 15(4), 762-766.

Tartaj, P., Morales, M., González-Carreño, T., Veintemillas-Verdaguer, S., \& Serna, C. (2005). Advances in magnetic nanoparticles for biotechnology applications. Journal of Magnetism and Magnetic Materials, 290, 28-34.

Wulff-Pérez , M., Martín-Rodriguez, A., Gálvez-Ruiz, M., \& de Vicente, J. ( 2013 ). The effect of polymer surfactant on the rheological properties of nanoemulsions. Colloid and Polymer Science, 291, 709-716.

Zamora Mora, V., Soares, P., Echeverria, C., Hernández , R., \& Mijangos, C. (2015). Composite chitosan/Agarose ferrogels for potential applications in magnetic hyperethermia. Gels., 1, 69-80.

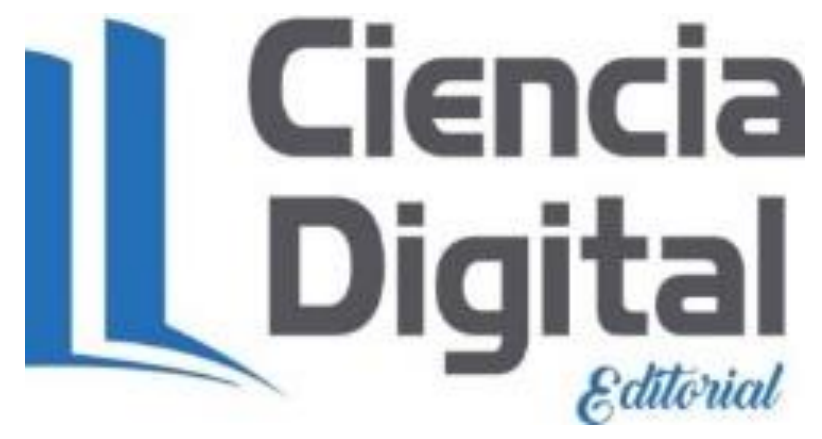


Para citar el artículo indexado.

Ormaza R., Coello J. \& Basantes E. (2019). Control del tamaño de microfibras magnéticas poliméricas durante el proceso de síntesis. Revista electrónica Ciencia Digital 3(1), 107-117. Recuperado

desde: http://cienciadigital.org/revistacienciadigital2/index.php/CienciaDigital/article/view/273/65 $\underline{5}$

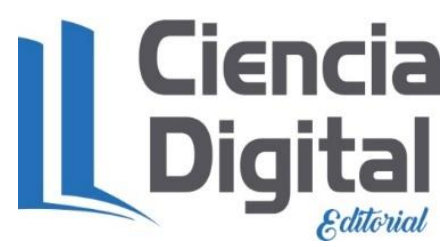

El artículo que se publica es de exclusiva responsabilidad de los autores y no necesariamente reflejan el pensamiento de la Revista Ciencia Digital.

El artículo queda en propiedad de la revista y, por tanto, su publicación parcial y/o total en otro medio tiene que ser autorizado por el director de la Revista Ciencia Digital.
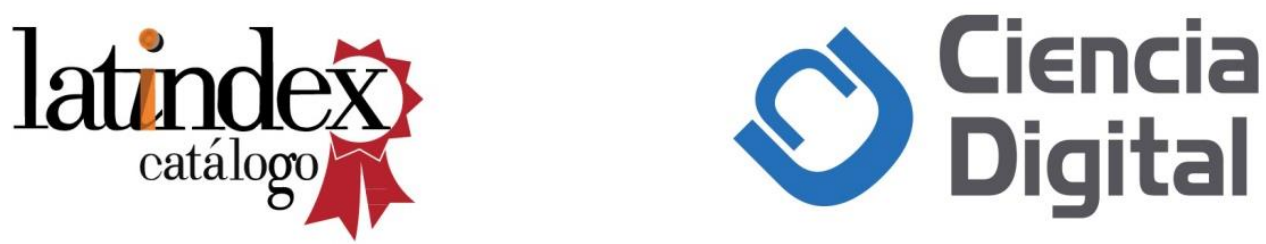